\title{
About the dynamics of the evection resonance
}

\author{
J. Frouard ${ }^{1,2}$, M. Fouchard ${ }^{1,2}$, and A. Vienne ${ }^{1,2}$ \\ 1 Institut de Mécanique Céleste et de Calcul des Ephémérides (IMCCE), Observatoire de Paris, UMR 8028, \\ Avenue Denfert-Rochereau, 75014 Paris, France \\ e-mail: frouard@imcce. fr \\ 2 Université de Lille 1, LAL-IMCCE, UMR 8028, 59000 Lille, France \\ e-mail: [Marc . Fouchard; Alain.Vienne] @univ-lille1.fr
}

Received 31 July 2009 / Accepted 10 November 2009

\begin{abstract}
Context. The evection resonance appears to be the outermost region of stability for prograde satellite orbiting a planet, the critical argument of the resonance indeed being found librating in regions surrounded only by chaotic orbits. The dynamics of the resonance itself is thus of great interest for the stability of satellites, but its analysis by means of an analytical model is not straightforward because of the high perturbations acting on the dynamical region of interest.

Aims. It is thus important to show the results and the limits inherent in analytical models. We use numerical methods to test the validity of the models and analyze the dynamics of the resonance.

Methods. We use an analytical method based on a classical averaged expansion of the disturbing function valid for all eccentricities as well as numerical integrations of the motion and surfaces of section.

Results. By comparing analytical and numerical methods, we show that aspects of the true resonant dynamic can be represented by our analytical model in a more accurate way than previous approximations, and with the help of the surfaces of section we present the exact location and dynamics of the resonance. We also show the additional region of libration of the resonance that can be found much closer to the planet due to its oblateness.
\end{abstract}

Key words. celestial mechanics - planets and satellites: general

\section{Introduction}

The problem of the stability of the orbits for real Solar System objects is a well-known topic and has been thoroughly studied by using many different dynamical models. Concerning the satellites of planets, an important problem is to determine the orbital stability limit below which the motion is bound to the planet,and above which the escapes of satellites on heliocentric orbits eventually occur. In the framework of the restricted circular threebody problem, the well-known Jacobi constant allows us to derive useful limits to bounded motion (see for example Murray \& Dermott 1999), but for prograde orbits it can be shown that stable regions subsist outside these limits due to the so-called evection resonance. A detailed knowledge of the dynamics induced by this resonance is therefore interesting for objects orbiting far away from the planet, such as the irregular (outer) satellites of the giant planets.

In their description of the lunar theory, Brouwer and Clemence (1961) present the evection term in $\cos \left(2 \lambda^{\prime}-2 \varpi\right), \varpi$ being the longitude of pericentre of the satellite and $\lambda^{\prime}$ the longitude of the perturbing body, which appears in the development of the solar disturbing function as the "largest periodic correction in the Moon's longitude" and the resonance have been of first importance to the dynamic of the moon in the past (Touma $\&$ Wisdom 1998). For distant orbits such those of the irregular satellites of the giant planets, this correction is crucial, as shown by recent analytic models (Yokoyama et al. 2003; Ćuk \& Burns 2004; Beaugé \& Nesvorný 2007). The dynamics of the resonance itself was studied for the first time by Hénon in his numerical study of the restricted three-body problem (Hénon 1969, 1970) where he shows that in the Hill's approximation, this resonance appears as a bifurcation of a family of simple periodic orbits (named " $g$ " in his papers) at a value of semi-major axis of $a=0.45 r_{\mathrm{h}}$ where $r_{\mathrm{h}}$ is the Hill's radius of the planet. However, this feature was not found in these studies for retrograde orbits. Hamilton \& Krivov (1997) studied the orbital motions of distant satellites using a generalized Tisserand constant and found the basic features of evection: resonant prograde orbits are elongated toward the perturbing body and their model shows in polar coordinates $(e \cos \alpha, e \sin \alpha)$ where $\alpha=\varpi-\lambda^{\prime}$ is the argument of the resonance, a characteristic eight-shape centered on $e=0$. This specific dynamic appears at a value of semi-major axis of $a=0.53 r_{\mathrm{h}}$, and exhibits two elliptic points at $\alpha=0$ and $\alpha=\pi$.

Retrograde orbits are shown to be elongated perpendicular to the perturber's direction, but the eight-shape observed once again in this direction was thought to be an artefact of the method by the authors. The importance of the evection resonance on the stability of satellites was pointed out by Nesvorny et al. (2003), where it is shown that the effects of the perturber's tides on a satellite in the resonance can accumulate at each aphelion passage and induce a important drift of the orbits, potentially causing the escape of the body.

The dynamics of the resonance was studied with an analytic model using an expansion of the disturbing function for the first time by Yokoyama et al. (2008). The "eight-shape" of the resonance for the planar problem was recovered by the authors, both 
for the prograde and retrograde case, and the apparition of the resonance was derived as $0.529 r_{\mathrm{h}}$ and $0.6933 r_{\mathrm{h}}$ for the prograde and retrograde case respectively.

However, numerically, resonant orbits can be found closer to the planet than predicted by these analytical models and do not seem to follow exactly the eight-shape found by previous authors. The aim of this paper is, first, to show and explain the limits associated with analytical models of the resonance, and secondly to resort to numerical methods, with the aim of localizing and studying the resonance itself. Since we are interested in the stability of the irregular satellites of Jupiter, the results of this paper will be applied in this context. We provide also an additional result concerning the dynamics of the resonance obtained when one takes into account the oblateness of the parent planet in the analytical model. Unlike the distant satellite orbits studied throughout this paper, we show that this modification only affects orbits very close to the planet.

In the following section, we construct and use an analytical model of the resonance and show the results that can be obtained. In Sect. 3, we resort to numerical studies (surfaces of section) to precisely localize the resonance and compare the results with those provided by the analytical model. In Sect. 4 we show how the oblateness of the parent planet affects the dynamics of the resonance. Finally, we present our conclusions and outline future work that should be performed in our last section.

\section{Analytical model}

To determine the limits of the use of an analytical model, we follow the method outlined by Yokoyama et al. (2008) and construct an analytical model of the resonance based on a development of the disturbing function in $\left(\frac{r}{r_{\odot}}\right)$. In addition we use an alternative semi-numerical method to verify the results.

\subsection{Development of the disturbing function in Legendre polynomials}

As in Yokoyama et al. (2008), we use a development of the disturbing function using Legendre polynomials. We consider in all the following calculations in this paper the motion of a satellite orbiting around a planet and perturbed by the Sun (indicated by subscript $\odot$ ) in a planetocentric system. The reference plane is the orbital plane described by the Keplerian motion of the Sun around the planet. The disturbing function $R_{\odot}$ related to the Sun of mass $m_{\odot}$ acting on the satellite's motion can be written using a Legendre polynomials development

$R_{\odot}=\frac{k^{2} m_{\odot}}{r_{\odot}} \sum_{l=2}^{\infty}\left(\frac{r}{r_{\odot}}\right)^{l} P_{l}(\cos (S))$,

using the Legendre polynomials

$$
\begin{aligned}
& P_{2}(u)=\frac{1}{2}\left(3 u^{2}-1\right), \\
& P_{3}(u)=\frac{1}{2}\left(5 u^{3}-3 u\right), \\
& P_{4}(u)=\frac{1}{8}\left(35 u^{4}-30 u^{2}+3\right), \\
& P_{5}(u)=\frac{1}{8}\left(63 u^{5}-70 u^{3}+15 u\right), \\
& P_{6}(u)=\ldots
\end{aligned}
$$

Here $k$ is the Gaussian gravitational constant and $S$ denotes the angle between the radius vectors of the satellite and the
Sun in the planetocentric frame. The $\cos (S)$ term in the Eq. (1) can be expanded using the cartesian coordinates of the bodies $\left(x, y, z, x_{\odot}, y_{\odot}, z_{\odot}\right), r$ and $r_{\odot}$ being the norm of the radius vectors

$$
\cos (S)=\frac{x}{r} \frac{x_{\odot}}{r_{\odot}}+\frac{y}{r} \frac{y_{\odot}}{r_{\odot}}+\frac{z}{r} \frac{z_{\odot}}{r_{\odot}} .
$$

Using the elliptical elements defined by $f$ the true anomaly, $\omega$ the argument of pericenter, $\Omega$ the longitude of node, and $I$ the inclination, one has

$$
\begin{aligned}
& \frac{x}{r}=\cos (\Omega) \cos (\omega+f)-\sin (\Omega) \sin (\omega+f) \cos (I), \\
& \frac{y}{r}=\sin (\Omega) \cos (\omega+f)+\cos (\Omega) \sin (\omega+f) \cos (I), \\
& \frac{z}{r}=\sin (\omega+f) \sin (I),
\end{aligned}
$$

and similar expressions for $\frac{x_{\odot}}{r_{\odot}}, \frac{y_{\odot}}{r_{\odot}}$, and $\frac{z_{\odot}}{r_{\odot}}$.

\subsection{Comparison in the order of Legendre polynomials}

Since the polynomials appear as powers of $\left(\frac{r}{r_{\odot}}\right)$ in the Eq. (1) and due to the (presumed) high distance in semi-major axis of the resonance from the planet, we must study the validity of the approximation's order before using any analytical result. To make a clear statement about the approximations made by using different orders of the polynomials with respect to the real motion, in Fig. 1 we represent stability maps for a Jovian satellite in the planar problem with the initial conditions $\lambda(0)=\varpi(0)=\lambda_{\odot}(0)=0$, the initial semi-major axis and eccentricity being chosen in the ranges $a \in[0.1: 0.2]$ AU and $e \in[0: 1$ [ respectively. We take as constants the semi-major axis of the Sun $a_{\odot}=5.202 \mathrm{AU}$ and the mass of Jupiter $m_{\text {jup }}=\frac{1}{1047.56}$. For each orbit, the total integration time $\tau$ was divided in two consecutive samples $\tau_{a}$ and $\tau_{b}$ of the same length and the maximal eccentricity $e_{\max }$ reached by the satellite for each sample was determined. The grey color code is given by $\frac{e_{\max }^{\tau_{b}}-e_{\max }^{\tau_{a}}}{e_{\max }^{\tau_{b}}}$, which is a stability criterion allowing the detection of chaotic and resonant orbits with respect to the regular ones (see Morbidelli 2002, for a discussion). White regions indicate initial orbits that have escaped the Hill's sphere of the planet and the grey scale indicates stable orbits in black and chaotic ones in grey levels (resonant orbits, despite their stability, are also highlighted in grey levels in the figures). Since we are interested in the stability of the outer satellites, these maps are of great importance and allow the easy location of the various resonances and chaotic zones. The integration was performed simulating the motion with a 2 nd order Legendre polynomial solely (namely the quadrupole term), then adding the $3 \mathrm{rd}$ order Legendre polynomial (the octupole term) and finally the full equations of motion.

By observing the differences, at the limit of stability, between the orbits in the three maps, we conclude from Fig. 1 that the 2 nd order Legendre development is unable to correctly reproduce the dynamics at the limit of stability given by the full equations of motion. We note that in these maps, the evection resonance is represented by the stable zone detached from the large one, surrounded by escaping regions and ranging from $a=0.145 \mathrm{AU}$ to $a=0.17 \mathrm{AU}$. On the other hand, the 3rd order development appears to be sufficient to approximate the real motion. We note that the 2 nd order development can still be used as a starting basis for studying the long-term behaviour of real satellites (see Ćuk \& Burns 2004), since these objects are closer to the planet. 
J. Frouard et al.: About the dynamics of the evection resonance
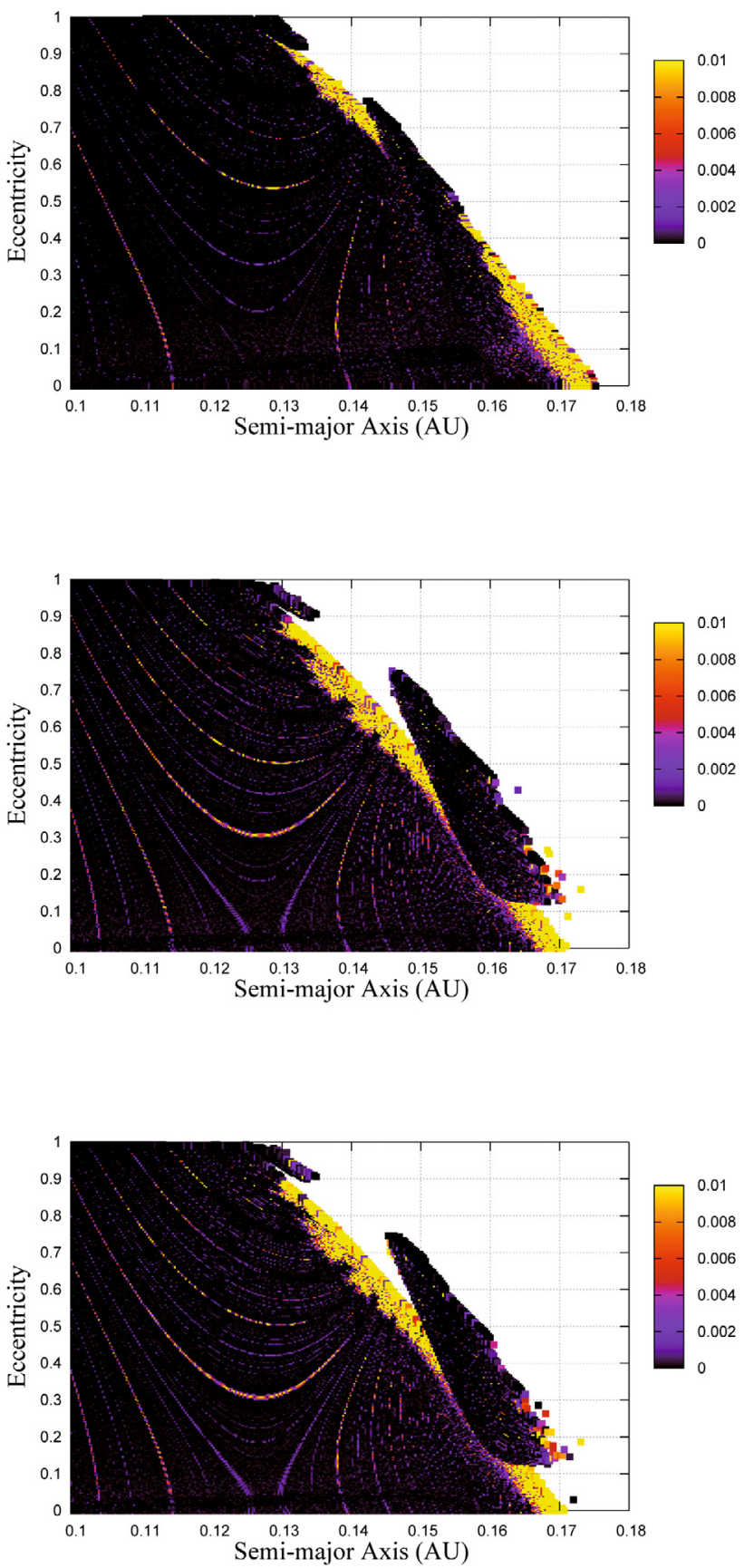

Fig. 1. Stability maps for a prograde Jovian satellite for $\tau=1000$ years using a 2nd order Legendre development (top), 3rd order Legendre development (middle), and the full equations of motion (bottom). See text for comments.

\subsection{The planar restricted three-body circular problem}

In the planar case, we automatically assume that $I=I_{\odot}=0$ thus $z=z_{\odot}=0$. The Sun's orbit is chosen to be circular, thus $e_{\odot}=0$ and $\omega_{\odot}=0$, implying that $f_{\odot}=\lambda_{\odot}, \lambda_{\odot}$ being the mean longitude of the Sun. Since an analytical model based solely on the 2nd order Legendre polynomial is insufficient for our purpose, we extend the method of Yokoyama et al. (2008) to the third order.

\subsubsection{Development and averaging}

The reduction to the planar case, with the perturber on a circular orbit, of Eq. (2) infers that $\cos (S)=\cos \left(f-\lambda_{\odot}+\varpi\right)$ if we consider a prograde satellite orbit, implying that $\varpi=\omega+\Omega$.
Considering first the 2nd order Legendre polynomial, the disturbing function is

$R_{2}=\frac{k^{2} m_{\odot} a^{2}}{2 a_{\odot}^{3}}\left(\frac{r}{a}\right)^{2}\left[\left(3 \cos ^{2}(S)-1\right)\right]$.

Since we are interested only in the critical argument of the evection resonance ( $\varpi-\lambda_{\odot}$ ), we average $R_{2}$ according to the mean anomaly $M$ of the satellite $:\left\langle R_{2}\right\rangle=\frac{1}{2 \pi} \int_{0}^{2 \pi} R_{2} \mathrm{~d} M$.

This averaging is performed using the exact closed-form equations of the two-body problem : $\left\langle\left(\frac{r}{a}\right)^{2}\right\rangle=1+\frac{3}{2} e^{2}$, $\left\langle\left(\frac{r}{a}\right)^{2} \cos (2 f)\right\rangle=\frac{5}{2} e^{2}$ and $\left\langle\left(\frac{r}{a}\right)^{2} \sin (2 f)\right\rangle=0$.

By using these equations, we obtain an averaged expression of the disturbing function that is valid for all values of the eccentricity of the satellite, resulting in

$\left\langle R_{2}\right\rangle=\frac{k^{2} m_{\odot} a^{2}}{2 a_{\odot}^{3}}\left[\frac{1}{2}\left(1+\frac{3}{2} e^{2}\right)+\frac{15}{4} e^{2} \cos \left(2 \varpi-2 \lambda_{\odot}\right)\right]$.

This is identical to Eq. (3.4) of Yokoyama et al. 2008.

If we consider a development in Legendre polynomials of up to order 3 , the corresponding expansion is

$R_{3}=R_{2}+\frac{k^{2} m_{\odot} a^{3}}{2 a_{\odot}^{4}}\left(\frac{r}{a}\right)^{3}\left[\left(5 \cos ^{3}(S)-3 \cos (S)\right)\right]$,

where it is necessary to expand and average the expressions $\left(\frac{r}{a}\right)^{3} \cos ^{3}(S)$ and $\left(\frac{r}{a}\right)^{3} \cos (S)$.

To this effect, we use the following averaging equations calculated with an algebraic manipulator :

$$
\begin{aligned}
\left\langle\left(\frac{r}{a}\right)^{3} \cos (3 f)\right\rangle & =-\frac{35}{8} e^{3}, \\
\left\langle\left(\frac{r}{a}\right)^{3} \sin (3 f)\right\rangle & =0, \\
\left\langle\left(\frac{r}{a}\right)^{3} \cos (f)\right\rangle & =-\frac{5}{2} e-\frac{15}{8} e^{3}, \\
\left\langle\left(\frac{r}{a}\right)^{3} \sin (f)\right\rangle & =0 .
\end{aligned}
$$

The final expression for the averaged expression is

$$
\begin{gathered}
\left\langle R_{3}\right\rangle=\left\langle R_{2}\right\rangle+\frac{k^{2} m_{\odot} a^{3}}{2 a_{\odot}^{4}}\left[\frac{3}{4}\left(-\frac{5}{2} e-\frac{15}{8} e^{3}\right) \cos \left(\varpi-\lambda_{\odot}\right)\right. \\
\left.-\frac{175}{32} e^{3} \cos \left(3 \varpi-3 \lambda_{\odot}\right)\right] .
\end{gathered}
$$

To study the dynamics of the resonance, we must place the problem in an integrable form, which can be achieved by choosing appropriate canonical variables within the Hamiltonian formalism of the problem. This is the aim of the next section.

\subsubsection{Canonical transformations of variables}

We place the problem in a Hamiltonian form by using the Delaunay variables $\left(L, G, L_{\odot}, l, g, l_{\odot}\right)$. The Delaunay variables are defined in the planar case to be

$$
\begin{aligned}
& L=\sqrt{\mu a}, \quad l=M \\
& G=\sqrt{\mu a\left(1-e^{2}\right)}, \quad g=\omega \equiv \varpi \\
& L_{\odot}, \quad l_{\odot}=M_{\odot} \equiv \lambda_{\odot} .
\end{aligned}
$$



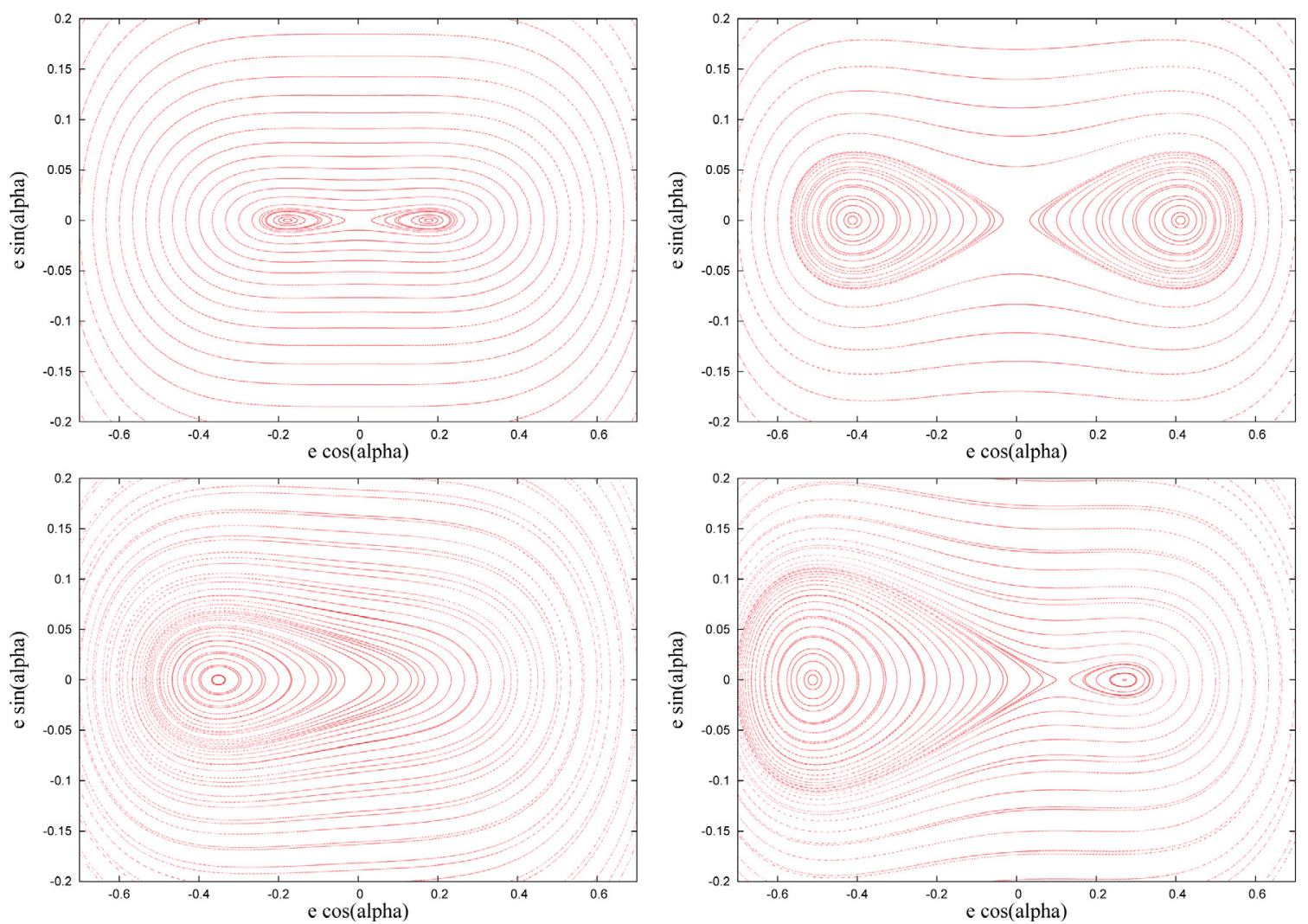

Fig. 2. Analytical averaged model. Dynamics of the evection resonance for a prograde satellite using a model up to the 2 nd order polynomial for $a=0.19 \mathrm{AU}$ (top left) and $a=0.2 \mathrm{AU}$ (top Right), and up to the 3rd order polynomial for $a=0.19 \mathrm{AU}$ (bottom left) and $a=0.2 \mathrm{AU}($ bottom right).

The Hamiltonian corresponding to a disturbing function of up to 2nd order is

$H_{2}=-\frac{\mu^{2}}{2 L^{2}}+n_{\odot} L_{\odot}-\left\langle R_{2}\right\rangle\left(L, G, L_{\odot}, g, l_{\odot}\right)$,

where $\mu=k^{2} m_{\text {jup }}$.

Our Hamiltonian in these variables is given by

$$
\begin{gathered}
H_{2}=-\frac{\mu^{2}}{2 L^{2}}+n_{\odot} L_{\odot}-\frac{k^{2} m_{\odot} L^{4}}{2 \mu^{2} a_{\odot}^{3}}\left[\frac{1}{2}+\frac{3}{4}\left(1-\left(\frac{G}{L}\right)^{2}\right)\right. \\
\left.+\frac{15}{4}\left(1-\left(\frac{G}{L}\right)^{2}\right) \cos \left(2 g-2 l_{\odot}\right)\right] .
\end{gathered}
$$

The above Hamiltonian has two degrees of freedom $\left(G, L_{\odot}, g, l_{\odot}\right)$. Indeed, since it is independant of $l$ the corresponding conjugate momenta $L=\sqrt{\mu a}$ is constant. The additive term $-\frac{\mu^{2}}{2 L^{2}}$ can thus be dropped from Eq. (9).

We then look for a canonical change of variables allowing us to reduce the Hamiltonian to one dimension, which is:

$\left(\begin{array}{cc}G & g \\ L_{\odot} & l_{\odot}\end{array}\right) \rightarrow\left(\begin{array}{ll}P_{1} & \alpha_{1} \\ P_{2} & \alpha_{2}\end{array}\right)$

with the generating function $S\left(P_{1}, P_{2}, g, l_{\odot}\right)=\left(g-l_{\odot}\right) P_{1}+l_{\odot} P_{2}$, which implies that

$P_{1}=G \quad, \quad \alpha_{1}=g-l_{\odot}$

$P_{2}=L_{\odot}+G \quad, \quad \alpha_{2}=l_{\odot}$.

In these variables, the momenta $P_{2}$ is constant and the term $n_{\odot} P_{2}$ can be suppressed from the Hamiltonian. The Hamiltonian in the resonant variables thus has one degree of freedom $\left(P_{1}, \alpha_{1}\right)$ and can be written by changing its sign to

$H_{2}=n_{\odot} P_{1}+\frac{k^{2} m_{\odot} a^{2}}{2 a_{\odot}^{3}}\left[\frac{-3}{4} \frac{P_{1}^{2}}{\mu a}+\frac{15}{4}\left(1-\frac{P_{1}^{2}}{\mu a}\right) \cos \left(2 \alpha_{1}\right)\right]$

(see Yokoyama et al. 2008).

Using the same method, we found the Hamiltonian corresponding to the expansion of the disturbing function up to third order

$$
\begin{aligned}
H_{3}=H_{2}+\frac{k^{2} m_{\odot} a^{3}}{2 a_{\odot}^{4}} & {\left[\frac{-15}{8}\left(1-\frac{P_{1}^{2}}{\mu a}\right)^{\frac{1}{2}}-\frac{45}{32}\left(1-\frac{P_{1}^{2}}{\mu a}\right)^{\frac{3}{2}} \cos \left(\alpha_{1}\right)\right.} \\
& \left.-\frac{175}{32}\left(1-\frac{P_{1}^{2}}{\mu a}\right)^{\frac{3}{2}} \cos \left(3 \alpha_{1}\right)\right]
\end{aligned}
$$

Tests have shown that inclusion of the subsequent orders $(4,5, \ldots)$ in the expansion of the averaged disturbing function leads to negligible differences in the study of the resonance, confirming the results for the comparison of Sect. (2.2) which concerns the nonaveraged case. We note that the Hamiltonian defined in Eq. (11) can possibly gives values of librating $\alpha_{1}$ other than those presented in the following : however their corresponding momenta $P_{1}$ are found to be negative, an impossibility for physical values of $a$ and $e$ (since $P_{1}=G=\sqrt{\mu a\left(1-e^{2}\right)}$ ), so we restrict our study to $P_{1} \geqslant 0$. In additional, $P_{1}(e)$ (with constant $a$ ) is bijective only in this case and we can show the dynamics of the model using the coordinates $\left(e, \alpha_{1}\right)$ in spite of the coordinates $\left(P_{1}, \alpha_{1}\right)$. In the following, $\alpha_{1}$ is denoted by $\alpha$ for simplicity.

In Fig. 2, we show the dynamical portraits of the resonance obtained with the 2 nd and the 3 rd order models for the prograde 

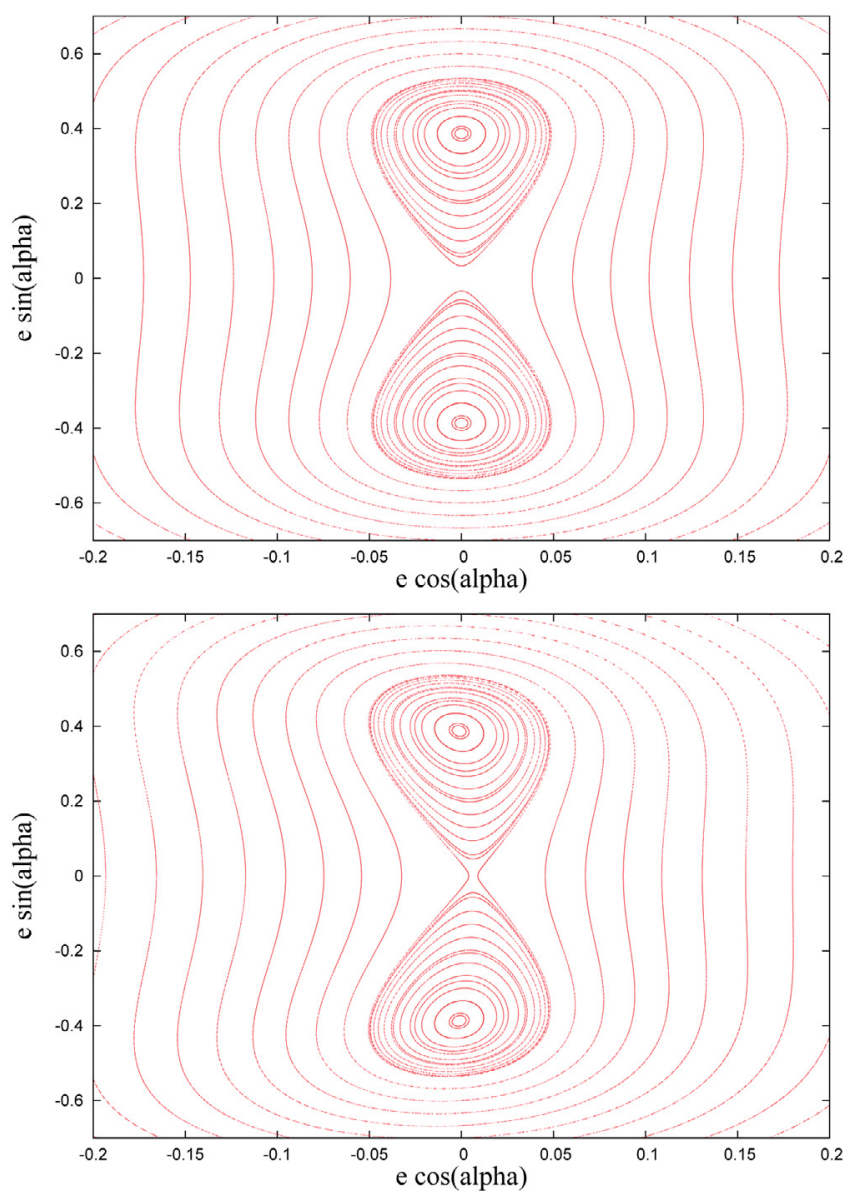

Fig. 3. Analytical averaged model. Dynamics of the evection resonance for a retrograde satellite using a 2nd order polynomial for $a=0.26 \mathrm{AU}$ (top), a 3rd order polynomial for $a=0.26 \mathrm{AU}$ (bottom).

case in the polar coordinates $(e \cos \alpha, e \sin \alpha)$ with $\alpha=\varpi-\lambda_{\odot}$. Despite the presence of two islands at $\alpha=0$ and $\alpha=\pi$ for the two approximations, we can discern a clear difference between their sizes and their formations; the 2 nd order model predicts that the two islands appear at $a=0.1878 \mathrm{AU}$, while in the 3rd order model, one of the islands $(\alpha=\pi)$ appears at $a=0 \mathrm{AU}$ and the other $(\alpha=0)$ appears at $a=0.1976 \mathrm{AU}$.

For retrograde orbits, we follow the convention of Saha $\&$ Tremaine (1993) concerning the definition of retrograde elements in the expansion. In Fig. 3, we show the dynamical portraits of the resonance in the retrograde case. Following Yokoyama et al. (2008), using a 2nd order Legendre polynomial the resonance appears at $0.6933 r_{\mathrm{h}}=0.245 \mathrm{AU}$ and the libration islands move to $\alpha=\frac{\pi}{2}$ and $\alpha=\frac{3 \pi}{2}$. The 3rd order term slightly modifies the dynamics; the hyperbolic point now has a non-null eccentricity ( $e=0.0121$ for the semi-major axis in Fig. 3 ) with $\alpha=0$ and the libration centers move to increasing values of $|\alpha|$ with increasing semi-major axis $\left(\alpha= \pm 93.29^{\circ}\right.$ in Fig. 3).

The global behaviour of the resonance in terms of semimajor axis and eccentricity predicted by the analytical model up to order 3 is shown in Fig. 4. In the upper figure (prograde case), the location of the stable elliptic points (light curves) and their corresponding separatrixes (bold curves) for the two islands is plotted. The island $\alpha=\pi$ begins at the origin of the semi-major axis at $e=0$ and only its upper separatrix is plotted; the lower separatrix remains at $e=0$. The second island $\alpha=0$ start at $a=0.1976 \mathrm{AU}$. Its center of libration and upper separatrix have a similar behavior to that of the previous island, and its lower
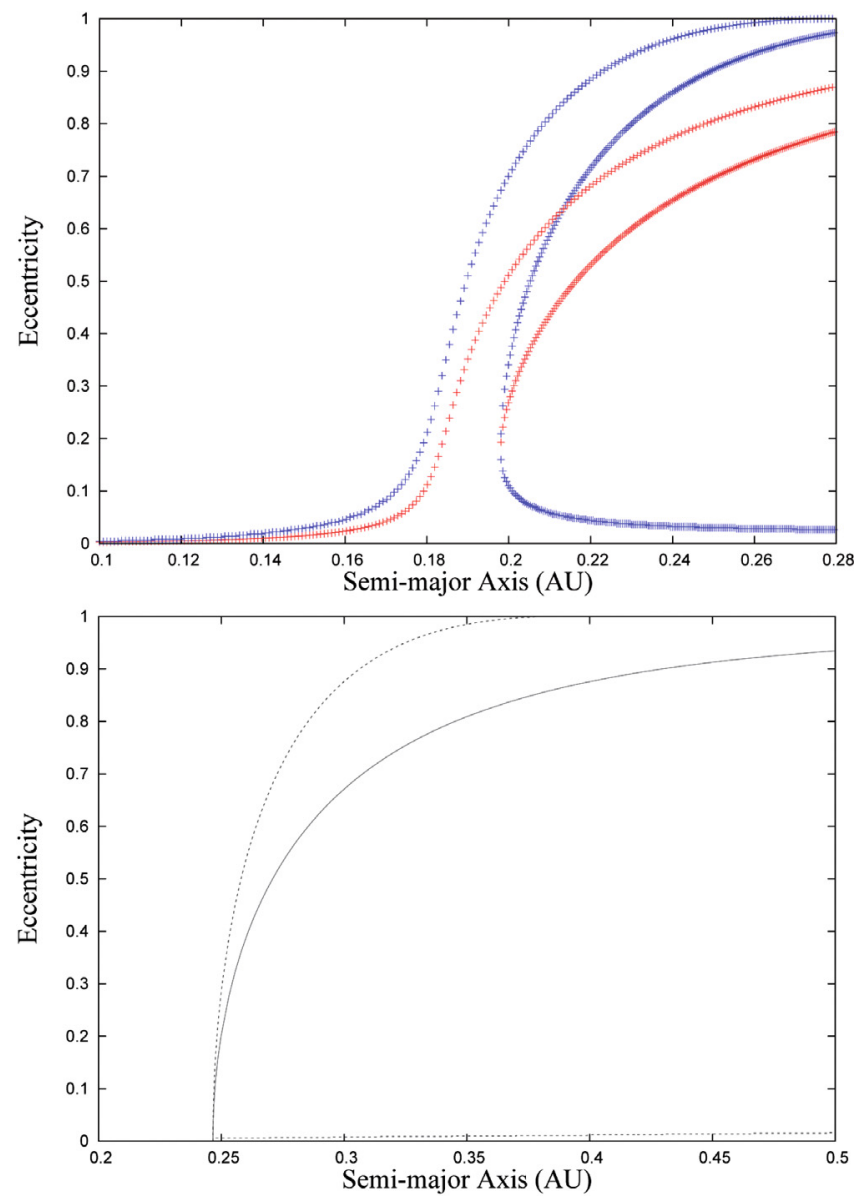

Fig. 4. Analytical averaged model. Localisation of the evection resonance for a prograde (top) and retrograde (bottom) satellite using the model up to the 3 rd order polynomial. Top: elliptic points (red curves), separatrixes and hyperbolic point (blue curves). Bottom: upper separatrix (upper curve), elliptic point (middle curve), hyperbolic point (bottom curve).

separatrix, which is the hyperbolic point of the resonance, decreases asymptotically to $e=0$ with increasing $a$. For the retrograde case (bottom figure), the upper separatrix and the elliptic point are indicated by the upper and middle curves respectively, and the hyperbolic point by the curve of very low eccentricity.

\subsection{Numerical averaging method}

To verify and expand the results obtained above using the analytical model, we use a numerical averaging procedure of the disturbing function. The disturbing function in cartesian coordinates $R\left(\boldsymbol{X}, \boldsymbol{X}_{\odot}\right)$, where $\boldsymbol{X}, \boldsymbol{X}_{\odot}$ represent the position vectors of the satellite and the Sun respectively, is expanded in elliptical elements $R\left(a, e, E, \varpi, a_{\odot}, \lambda_{\odot}\right)$, where $E$ denotes the eccentric anomaly of the satellite. The dependance on the longitude of the satellite is numerically averaged following the procedure of Moons (1994) (where the change of variables comes from the Kepler equation)

$\langle R\rangle=\frac{1}{2 \pi} \int_{0}^{2 \pi} R \mathrm{~d} M=\frac{1}{2 \pi} \int_{0}^{2 \pi}(1-e \cos E) R \mathrm{~d} E$.

The remaining angles are fixed to values corresponding to the evection resonance : $\varpi=\lambda_{\odot}, \varpi=\lambda_{\odot}-\pi$ (prograde case), and $\varpi=\lambda_{\odot}+\frac{\pi}{2}, \varpi=\lambda_{\odot}+\frac{3 \pi}{2}$ (retrograde case). We have shown that 

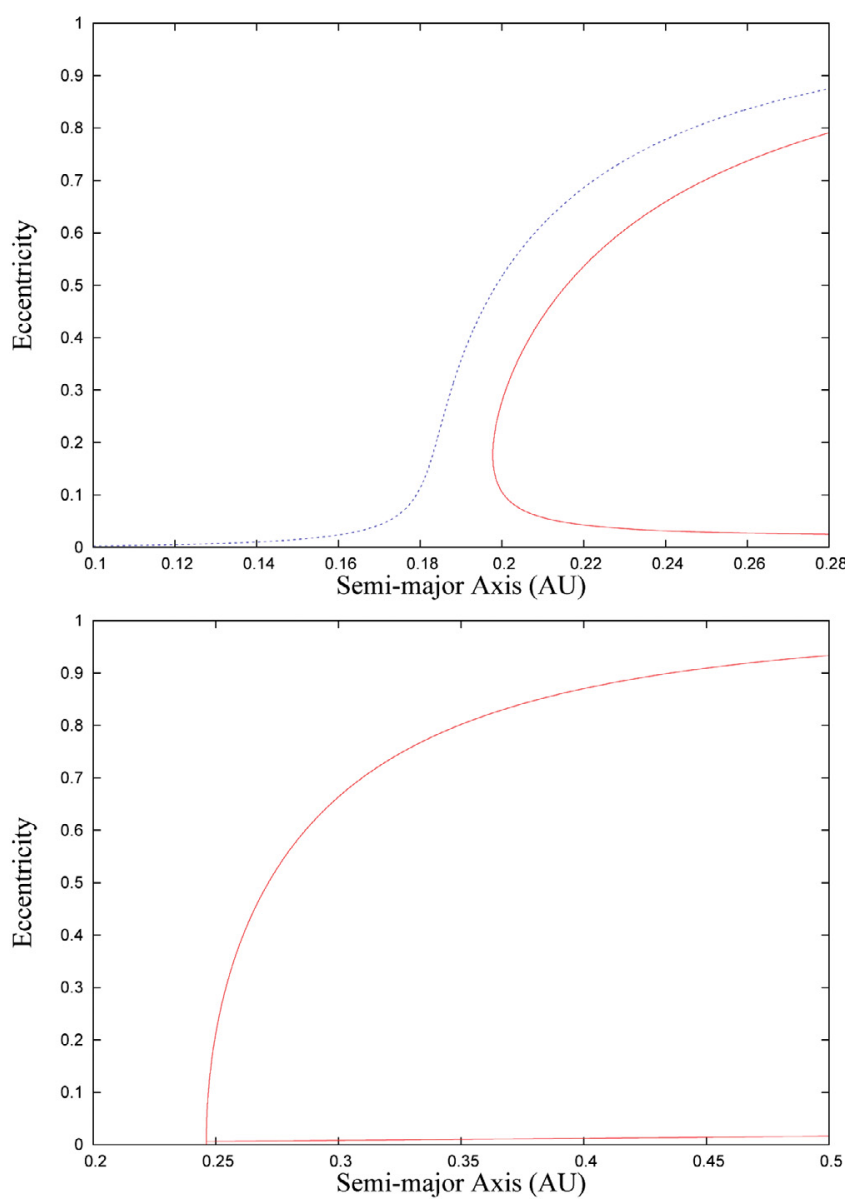

Fig. 5. Numerically averaged model. Localisation of the elliptic and hyperbolic points of the evection resonance for prograde orbits (top) and retrograde ones (bottom).

for retrograde orbits, the libration centers move slightly and $\alpha$ is no longer $\pm \frac{\pi}{2}$, but the difference in the localisation of the exact resonance is marginal, for example the error in $e$ is smaller than 0.01 for $a=0.5 \mathrm{AU}$. This method is applicable to all eccentricities and is not limited by the ratio $\frac{a}{a_{\odot}}$, in contrast to the classical analytic Legendre expansion. The frequency $\dot{\varpi}$ can then be obtained using the corresponding "secular form" of the Lagrange equation

$\dot{\varpi}=\frac{\sqrt{1-e^{2}}}{n a^{2} e} \frac{\partial\langle R\rangle}{\partial e}$,

which is calculated with a numerical partial derivative scheme and compared to the mean motion of Jupiter to obtain the elliptic and hyperbolic points of the resonance. The results represented in Fig. 5 show very good agreement with the stable and hyperbolic points given by the 3rd order analytical model, and the curves are merged with the corresponding ones of Fig. 4.

\subsection{Discussion}

We note several things about these results. The discrepancy between the dynamics caused by the 2 nd order and 3 rd order terms from the expansion of Eq. (1) is that despite being inferior in the ratio $\left(\frac{r}{r_{\odot}}\right)$ by an order of magnitude compared to the 2 nd order term, the 3 rd order polynomial therefore plays a major role due to the effect of strict $e$ in its formulation, comparing to the $e^{2}$ in the 2 nd order one. We note that the "eight-shape" resonant
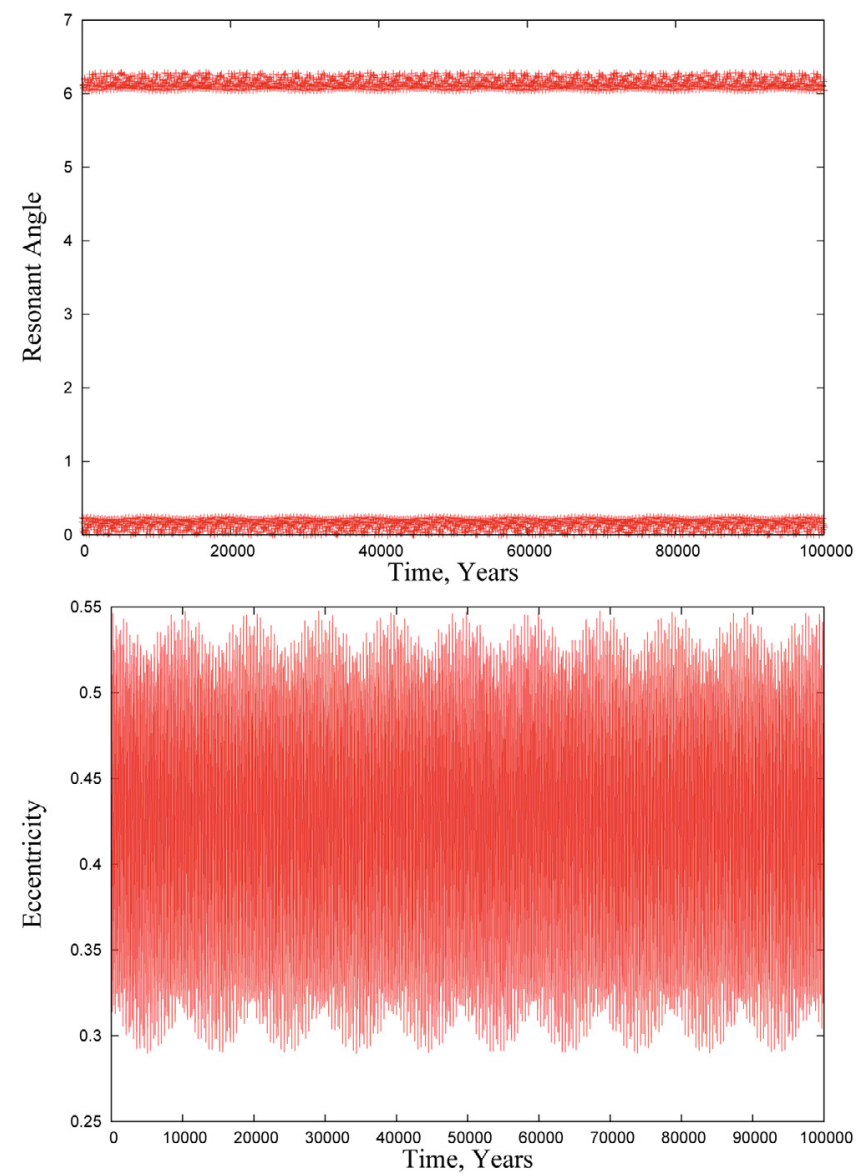

Fig. 6. Time evolution of the evection resonant angle (top) and eccentricity (bottom) for a satellite with initial elements $a=0.155 \mathrm{AU}$, $e=0.5$. The initial angles are set to zero.

dynamics was also found by Hamilton \& Krivov (1997), but the construction of their Generalized Tisserand constant was achieved using solely a 2 nd order Legendre polynomial to introduce the mean longitude of the Sun in their model. For the model used in this paper, we have shown that the following terms in the expansion (of order $>3$ in the ratio $\frac{r}{r_{0}}$ ) become negligible in the interval of semi-major axis studied and do not introduce noticeable changes, as shown by Fig. 5 .

The main consequence of inclusion of the 3rd order term is the loss of symmetry between the two islands in Fig. 2. The islands now have distinct evolutions, implying different sizes of the islands at given values of the semimajor axis. We note that the island $(\sigma=\pi)$ can now be found at all values of semi-major axis.

However, despite the precision provided by the 3rd order term, the analytical model still fails to describe the real dynamics, since one can find numerically resonant orbits at semi-major axis and eccentricity values inferior to those predicted by the model, as shown for example in Fig. 6. In Fig. 1, we however show that a non-averaged expansion up to the 3rd order term seems to be sufficient to correctly describe the true motion.

The discrepancies observed from the numerical experiments come from the fact that we are using a normal form given by Eq. (11), but without having explicitly written the corresponding generating function (see for example Ferraz-Mello 2007) which allows to determine the analytical relations between osculating and averaged variables. The initial osculating elements are thus considered to be equal to the averaged elements used in 
the analytical model. If this simplification works well in many cases where the perturbation is small, here at the limit of stability it is non-negligible (see a discussion in Yokoyama et al. 2003). To consider this problem, one could use a perturbation method that explicitly permits the calculation of the generating function, such as Lie series method. However, this applies to use an explicit development of the disturbing function in Fourier series to keep things simple, and thus introduces an expansion in eccentricity (as opposed to the averaging method used above that is valid for all eccentricities), which would limits the applications of the model for low to moderate excentricities. In the following, we use an alternative and more direct way by using surfaces of section to analyze the resonance. However, if the high perturbation prevents us from correctly use the analytical model, one can choose to apply this method to a region where it is "safe" as the one of Sect. 4. In this section, we investigate the evection resonance in a region much closer to the planet where the solar perturbation is small, taking into account the oblateness of the planet, and using the above analytical model.

In addition, as shown in the next section resonant retrograde orbits cannot be found numerically despite their existence being expected by the model. Here the problem originates from the fact that the retrograde resonance is localized in a large chaotic zone corresponding to escaping orbits.

\section{Surfaces of section}

To carefully study the planar problem, we take advantage of the two-dimensionality of the Hamiltonian of the problem expressed in the rotating frame evolving with the mean motion of Jupiter, and we use the Poincaré surface of section method (PSS). The corresponding Hamiltonian in the rotating frame (see for example Valtonen \& Karttunen 2006) is given by

$H=\frac{1}{2}\left(P_{x}^{2}+P_{y}^{2}\right)+P_{x} y-P_{y} x-\frac{1-\mu}{r_{1}}-\frac{\mu}{r_{2}}$,

where $x, y, P_{x}, P_{y}$ are, respectively, the coordinates and momenta of the particle in the rotating frame centered on the barycenter of the system, $\mu$ is the mass of the planet, $1-\mu$ is the mass of the Sun, and we have the distances

$r_{1}^{2}=(x+\mu)^{2}+y^{2}$,

$r_{2}^{2}=(x-(1-\mu))^{2}+y^{2}$.

The PSS is then constructed from the motion of the particle in the cartesian coordinates $(x, y, \dot{x}, \dot{y})$ by choosing a surface $S$ (for example $y=0)$ and plotting the remaining coordinates $(x, \dot{x})$ each time the particle crosses the surface $S$ with $\dot{y}>0$. The dynamics of different orbits can then be represented for the same value of the Jacobi constant $C$, which is the only integral of motion of the system,

$C=x^{2}+y^{2}+2\left(\frac{1-\mu}{r_{1}}+\frac{\mu}{r_{2}}\right)-\dot{x}^{2}-\dot{y}^{2}$.

We emphasize that the PSS corresponds to the complete perturbed motion of the particle as opposed to an analytical model constructed from an approximation. In Fig. 7, we show two stability maps integrated up to 1000 periods of Jupiter, corresponding to prograde orbits with the initial conditions $\lambda(0)=$ $\lambda_{\odot}(0)=\varpi(0)=0$ (pericenter in conjunction with the Sun), and $\lambda(0)=\lambda_{\odot}(0)=0, \varpi(0)=\pi$ (pericenter in opposition with the Sun). The upper map in Fig. 7 is similar to the last one of Fig. 1 but here we use the MEGNO as an indicator of chaos
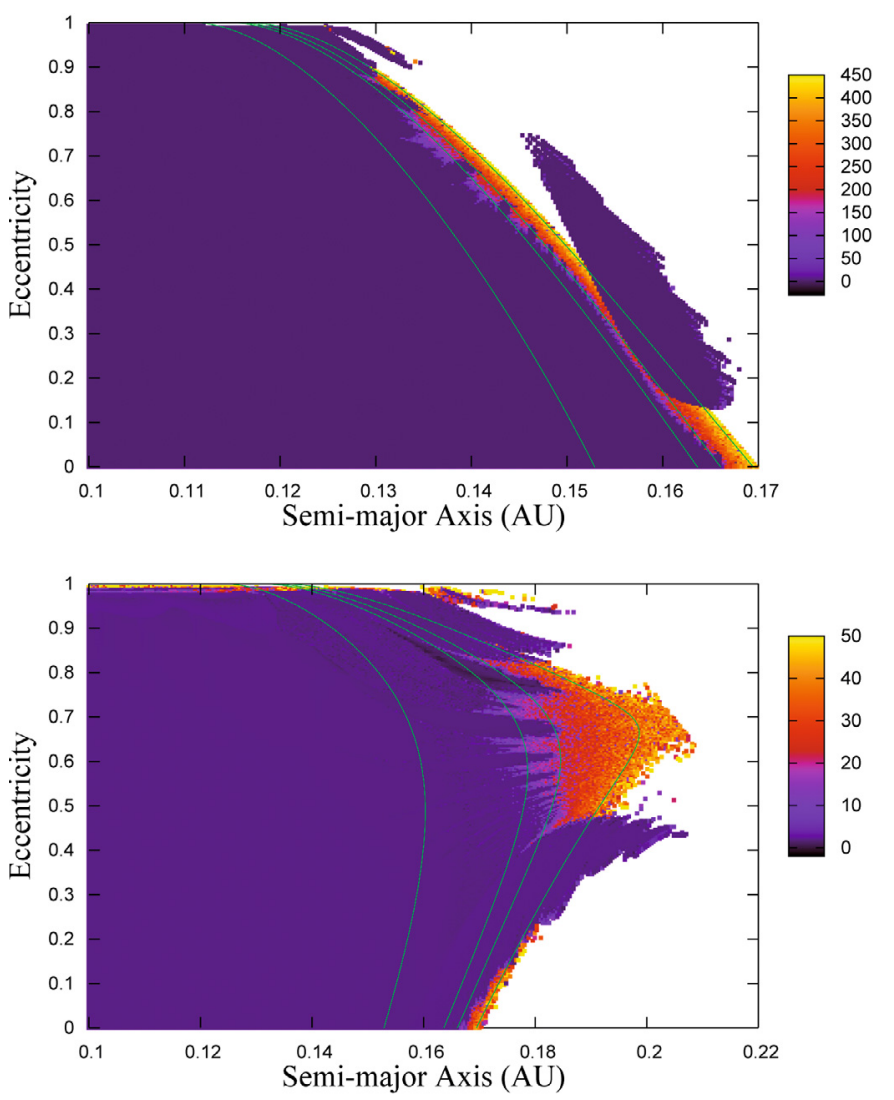

Fig. 7. Stability maps obtained with the MEGNO for a prograde Jovian satellite and 4 iso-Jacobian curves. Initial conditions: pericenter in conjunction with the Sun (top), pericenter in opposition to the Sun (bottom).

(Cincotta et al. 2003) as we have observed its qualities (Frouard et al. 2008). The MEGNO is a fast and reliable chaos indicator which uses the time evolution of a tangent vector computed from the variational equations of the motion. Apart from the detection of chaotic orbits, for ordered motions this indicator is able to clearly distinguish resonant orbits from non-resonant ones. In the maps of Fig. 7, the white regions indicate satellites that have escaped the Hill's sphere of Jupiter while having a hyperbolic orbit with respect to an elliptic Keplerian one. We identified the limit of stability for initial circular orbits at a semi-major axis of $a=0.17 \mathrm{AU}$ which is consistent with the value $a=0.48 r_{\mathrm{h}}$ found by Alvarellos (1996). Dark blue indicates stable orbits, while purple to yellow show different levels of chaoticicity for unstable orbits.

From the studies already cited (and our own numerical experiments), we know that the evection resonance in the prograde case has two libration islands, situated at $\alpha=0$ and $\alpha=\pi$. This explain the choice of the initial conditions for the stability maps, which are chosen to place the satellite in each island at the beginning of the integration. In the maps, we indicate four iso-Jacobian curves that correspond to the PSS showed in Fig. 8; curves from left to right in the maps have respectively the values $C=3.041,3.0395,3.0392$ and 3.0388. We can then explain the dynamic shown in the stability maps with the surfaces of section.

For the higher value of the Jacobi constant $(C=3.041)$, we are able to recognize at the center of the figure the orbit corresponding to the family of simple periodic orbits " $g$ " (Hénon $1969,1970)$ and the libration $\alpha=\pi$ is still not allowed. We

1 The periodic orbit named $g$ is the resonance with the critical argument $\lambda-2 \lambda_{\odot}+\varpi$ in the inertial frame. 

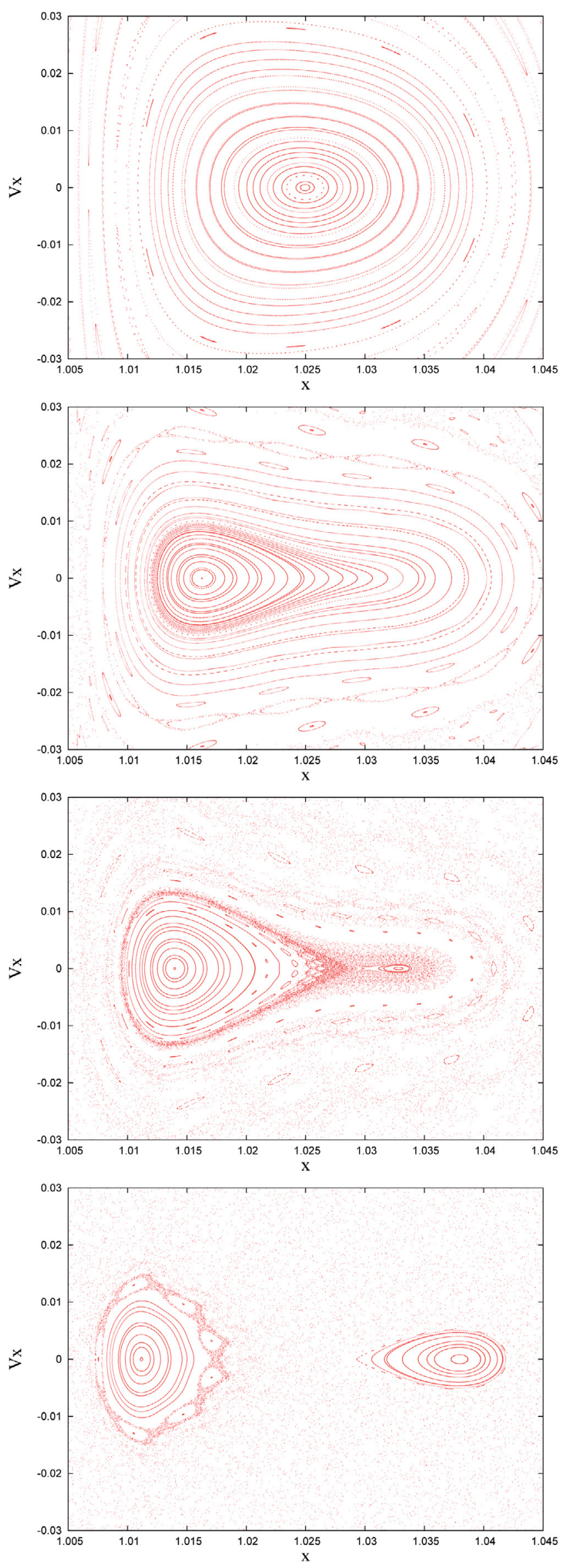

Fig. 8. From top to bottom: surfaces of section for $C=3.041, C=$ $3.0395, C=3.0392$, and $C=3.0388$. determined its appearance at $C=3.0402552$. For $C=3.0395$ (the second figure), we can see that several low-order resonances appear along with chaos due to the overlapping between them and the "spreading" of their separatrixes. The periodic orbit $g$ (now the evection resonance $\alpha=\pi$ ) has moved to lower $x$. We can see a good correspondence with the stability maps of Fig. 7, where the corresponding Jacobian curves is in the weak chaotic region. For $C=3.0392$, the hyperbolic point of the resonance was created and corresponds to the "critical orbit" " $g_{1}$ " in Hénon (1970), and produced the second island centered on $x=1.033$, which corresponds to the evection argument $\alpha=0$ (we found the appearance of the island at $C=3.0392409$ ). In Hénon (1970), the island corresponding to the original orbit $g$ was divided at its center by the critical orbit $g_{1}$, creating two libration islands of similar size whose centers are the elliptic orbits $g^{\prime}$. This is not the case here, where the bifurcation has created an island of a very small size comparing to the other. This difference can be caused by the Hill's approximation not being used in this paper. For the same value of $C$ we can observe that after the bifurcation, the chaos related to the hyperbolic point is still confined, but is about to be mixed with the already present one, giving a nearly generalized chaos on the surface of section. Obviously, we observe this phenomenon on the stability maps, where the iso-Jacobian curve now passes through stable resonant and strongly chaotic zones. Quasi-periodic orbits survive only at high eccentricities. The map also shows, in accordance with the surface of section, that the iso-Jacobian curve passes through the detached stability zone, which corresponds to the newly created island. Finally, for $C=3.0388$ (last surface of section), a value slightly higher than the value corresponding to the Lagrangian point $L_{1}\left(C_{L_{1}}=3.0387559\right)$, thus just before the possible escape of the particle, the chaos is generalized.

\subsection{Discussion}

We mention that the two islands are very sensitive to the initial conditions that we choose for the PSS; the motion will be resonant in one of the islands if the resonant angle $\alpha$ at the beginning of the integration is very close to one of the exact values of the resonance (e.g., $\alpha=0$ or $\alpha=\pi$ ). In fact we can see in Figs. 7 and 8 that the islands are surrounded only by chaotic regions.

Can we draw conclusions about the analytical model with the help of the surfaces of section presented above? Figs. 2 and 8 cannot be rigorously compared because each of the four PSS correspond to a single value of the Jacobi constant, which implies that the orbits integrated in one PSS have different initial elements $a$ and $e$ with $\varpi=0$ or $\varpi=\pi$ (the particle always starts in the Sun-Jupiter line, with a perpendicular velocity, thus the initial $\lambda$ and $\lambda_{\odot}$ are the same for all orbits). Despite this and the fact that different coordinates are used in Figs. 2 and 8, the similarity between the PSS and the dynamic given by the analytical model up to the 3rd order is striking. On the other hand, the surfaces of section obtained with the Hill's approximation (see Hénon 1970) are similar to the dynamics predicted by the analytical model up to the 2nd order. The main difference caused by the PSS is that the island $\alpha=\pi$ is created at a specific value of $C$; the central periodic orbit for $C \geqslant 3.0402552$ (see the upper graph in Fig. 8) corresponds to the periodic orbit $g$.

The evection for retrograde orbits cannot be found by means of surfaces of section. The family of simple periodic orbits " $f$ " in Hénon (1970) indeed experiences no bifurcation. If orbits can numerically be found librating around the centers $\alpha=\frac{\pi}{2}$ and 

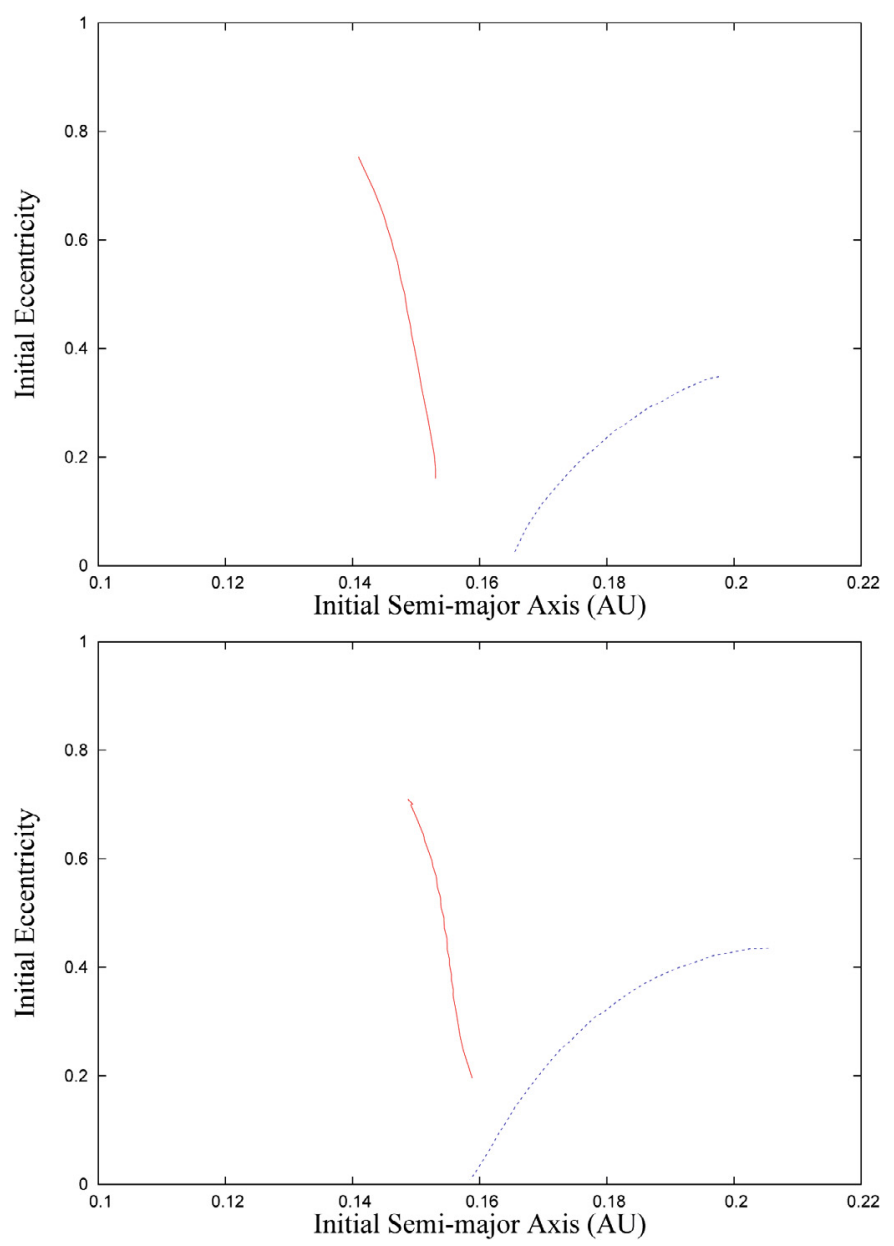

Fig. 9. Location of the evection resonance with the PSS in initial osculating elements. Top: $\lambda_{\odot}(0)=0, \lambda(0)=\pi$. Left curve: libration $\alpha=\pi$ with $\varpi(0)=\pi$, right curve: libration $\alpha=0$ with $\varpi(0)=0$. Bottom: $\lambda_{\odot}(0)=0, \lambda(0)=0$. Left curve: libration $\alpha=0$ with $\varpi(0)=0$, right curve: libration $\alpha=\pi$ with $\varpi(0)=\pi$.

$\alpha=\frac{3 \pi}{2}$, it is always temporarily, and they alternate between circulation and/or libration around the other elliptic point after a certain amount of time.

Figure 9 indicates the centers of libration given by the PSS for several initial osculating variables. Since we analyze the complete problem, the centers of the resonance are found to depend of the initial osculating variables and the use of a more sophisticated perturbation method than in Sect. 2 would have brought a similar phase dependency with respect to the initial variables. In the two figures, the left and right curves correspond to the librations $\alpha=\pi$ and $\alpha=0$ respectively. In Fig. 10, we present the location of the two islands of the resonance as given by the PSS in averaged elements, along with their counterparts found by the numerically averaged model (Sect. 2.4). The averaged elements were obtained by averaging the semimajor axis and the eccentricity of the orbits found by the PSS in Fig. 9 over 10000 years. In this figure, the two left curves correspond to the orbits found with the PSS, superimposed on those of Fig. 5. We can appreciate the interval between the curves found by the two methods, which illustrates the lack of precision of the analytical model and emphasizes that the high solar perturbation prevent us from using it. In the next section, we show that the analytical model can still be used if the solar perturbation is sufficiently weak, that is very close to the planet.

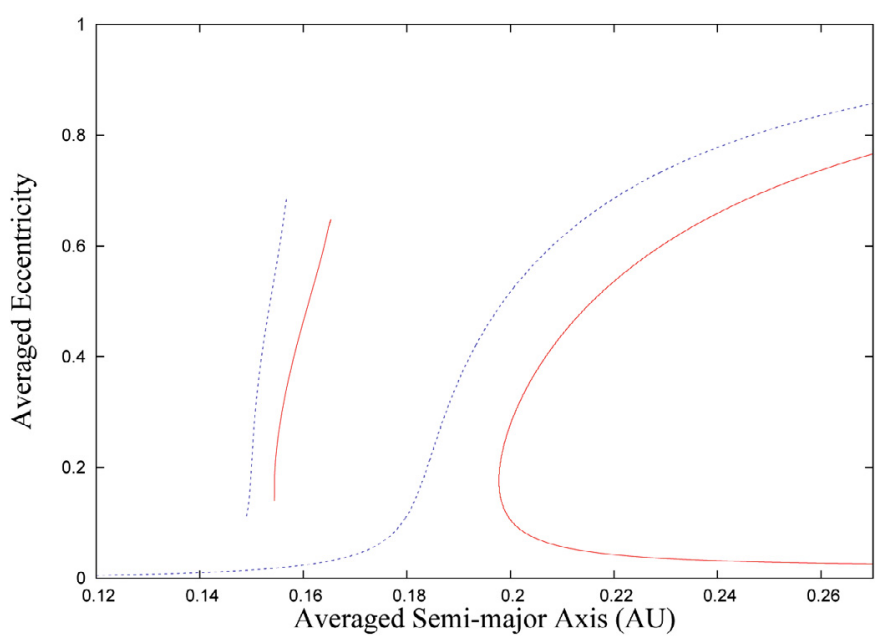

Fig. 10. Location of the evection resonance for prograde satellites with the PSS (in averaged elements) (the two left curves) and by the numerically averaged model (the two right curves). The island $\alpha=\pi$ is indicated by the dot line, the island $\alpha=0$ by the solid one.

\section{Evection resonance taking into account the oblateness of the planet}

To study the effect of the resonance very close to the planet, we start from the analytical model described in Sect. 2, and take into account the correction due to the second harmonic in the development of the potential of the planet. Indeed, close to the planet, the frequency of the pericenter of the satellite is insufficiently high to be comparable to the mean motion of the Sun, and does not allows the appearance of the evection resonance. The oblateness of the planet has the well-known effect of increasing the frequency of pericenter of a satellite. One can note that this effect is only local, and we have checked that no apparent change is present for the outer region discussed in the previous sections. We have also verified how the description of the solar perturbation could be improved by using different orders of approximation as in Sect. 2.2 and conclude that due to the small ratio $\left(\frac{a}{a_{\mathrm{o}}}\right)$, the 2 nd order polynomial alone is sufficient. To obtain the additional term that depends on the oblateness of the planet, we proceed in the following with a method similar to that used at the beginning of this paper.

\subsection{Development and averaging}

The gravitational potential of a planet formulated in spherical coordinates $(r, \lambda, \phi)$, where the axis of revolution of the body coincides with its rotation axis, is written, following Duriez (2002), as

$$
\begin{gathered}
U(r,-, \phi)=\frac{k^{2} m}{r}\left(1-J_{2} \frac{a_{\mathrm{e}}^{2}}{r^{2}} P_{2}(\sin (\phi))-J_{4} \frac{a_{\mathrm{e}}^{4}}{r^{4}} P_{4}(\sin (\phi))-\ldots\right. \\
\left.-J_{2 n} \frac{a_{\mathrm{e}}^{2 n}}{r^{2 n}} P_{2 n}(\sin (\phi))-\ldots\right),
\end{gathered}
$$

where $m$ is the mass of the planet, $a_{\mathrm{e}}$ is its equatorial radius, and the $J_{n}$ are the zonal harmonics. The $P_{n}$ are the Legendre polynomials defined in Sect. 2.1. This simplification limits the application of the model to planets with a sufficiently low value of obliquity $\epsilon$ to be considered negligible; this is the case for Jupiter $\left(\epsilon=3.12^{\circ}\right)$, Mercury $\left(\epsilon \sim 0.1^{\circ}\right)$, and Venus (retrograde rotation: $\left.\epsilon=177 \cdot 3^{\circ}\right)$. However, for the last two planets all values of 


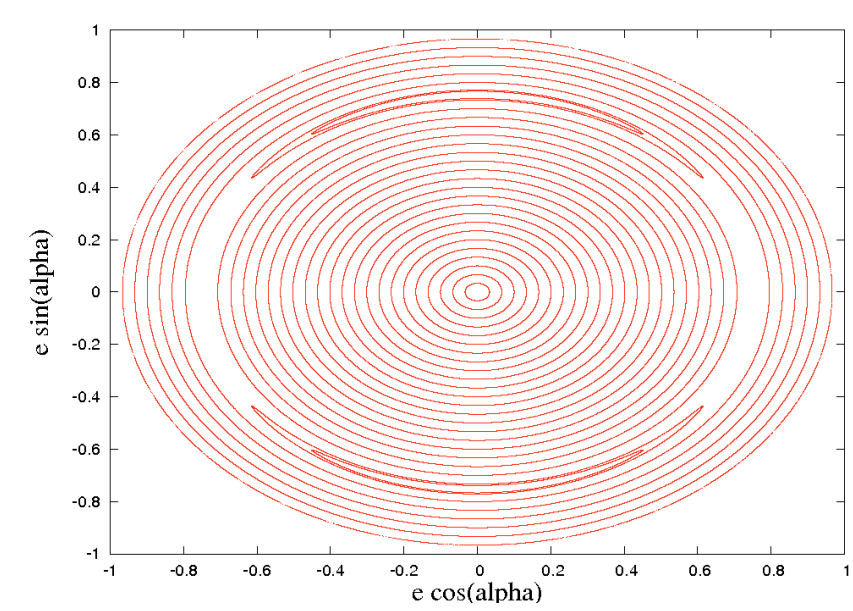

Fig. 11. Dynamical portrait of the evection resonance for a Jovian satellite with semi-major axis $a=0.00515 \mathrm{AU}$, taking into account the Jupiter's $J_{2}$.

eccentricity of the orbits affected by the resonance lead to pericenters that are inside the physical radius of the planet. For Jupiter, only the high-eccentricity part of the resonance induces this effect. The physical coefficients of Jupiter are $a_{\mathrm{e}}=$ $71398 \mathrm{~km}$ and $J_{2}=0.01475$. We note that the resonance for the cases of Mars and Earth was studied analytically by Breiter (2000). The perturbation caused exclusively by the 2 nd order Legendre polynomial, and involving only the $J_{2}$ coefficient is given by

$U_{J_{2}}=-k^{2} m J_{2} \frac{a_{\mathrm{e}}^{2}}{r^{3}}\left(\frac{3}{2} \sin ^{2}(\phi)-\frac{1}{2}\right)$.

Following classical calculations and using the averaging relation $\left\langle\left(\frac{a}{r}\right)^{3}\right\rangle=\left(1-e^{2}\right)^{\frac{-3}{2}}$ in a similar way to that in Sect. 2.3.1, we obtain the expression for $U_{J_{2}}$ averaged over the mean anomaly of the satellite :

$\left\langle U_{J_{2}}\right\rangle=k^{2} m J_{2} \frac{a_{\mathrm{e}}^{2}}{a^{3}}\left(\frac{1}{2}-\frac{3}{4} \sin ^{2}(i)\right)\left(1-e^{2}\right)^{-\frac{3}{2}}$.

\subsection{The planar case}

When $i=0$, Eq. (18) expressed in resonant variables becomes

$$
\left\langle U_{J_{2}}\right\rangle=\frac{1}{2} k^{2} m J_{2} \frac{a_{\mathrm{e}}^{2}}{a^{3}}\left(\frac{P_{1}^{2}}{\mu a}\right)^{\frac{-3}{2}} .
$$

The phase portrait of the resonance in polar coordinates for prograde orbits of semi-major axis $a=0.00515$ AU can be seen in Fig. 11 (retrograde resonant orbits were not found). The effect of the oblateness is to change the libration centers of the resonance by $\frac{\pi}{2}$. The resonance also appears to be narrower.

A numerical example of a resonant orbit integrated with the full equations of motion is shown in Fig. 12.

In Fig. 13, the exact resonance and its width are shown. The location of the averaged semi-major axis of the four main Jovian massive satellites (Io, Europe, Ganymede and Callisto) $)^{2}$ are also indicated by vertical lines. We note that the pericenter of the satellite's orbit is found to be inside the physical radius of Jupiter when the upper separatrix of the resonance exceed $a=0.01176$ AU for $e=0.959$. For the exact resonance, the critical values are $a=0.01628$ AU with $e=0.971$.

\footnotetext{
2 taken from http://ssd.jpl.nasa.gov/?sat_elem
}
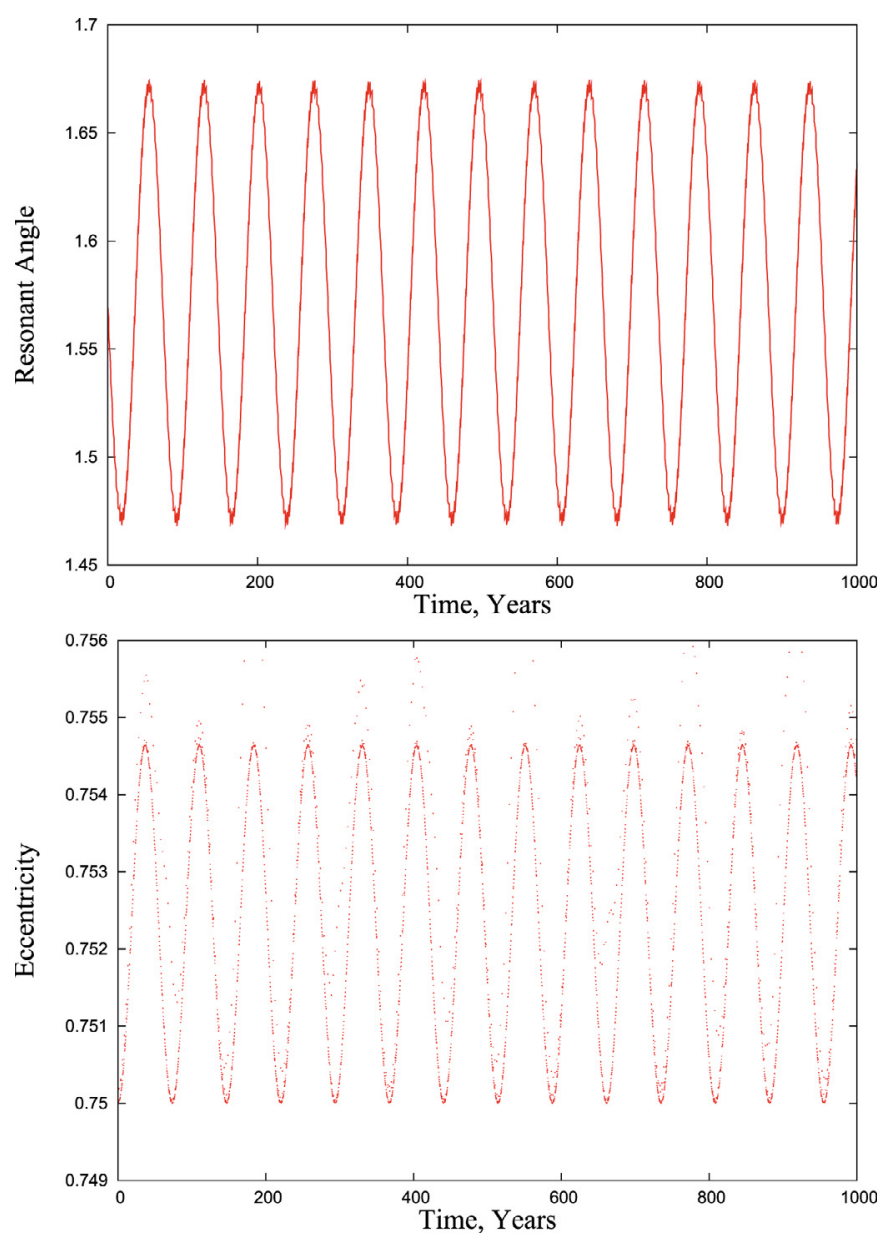

Fig. 12. Time evolution of the resonant angle (top) and eccentricity (bottom) of an orbit integrated with the full equations of motion with initial elements $a=0.00515 \mathrm{AU}, e=0.75, \lambda=0, \varpi=\frac{\pi}{2}$ and $\lambda_{\odot}=0$.

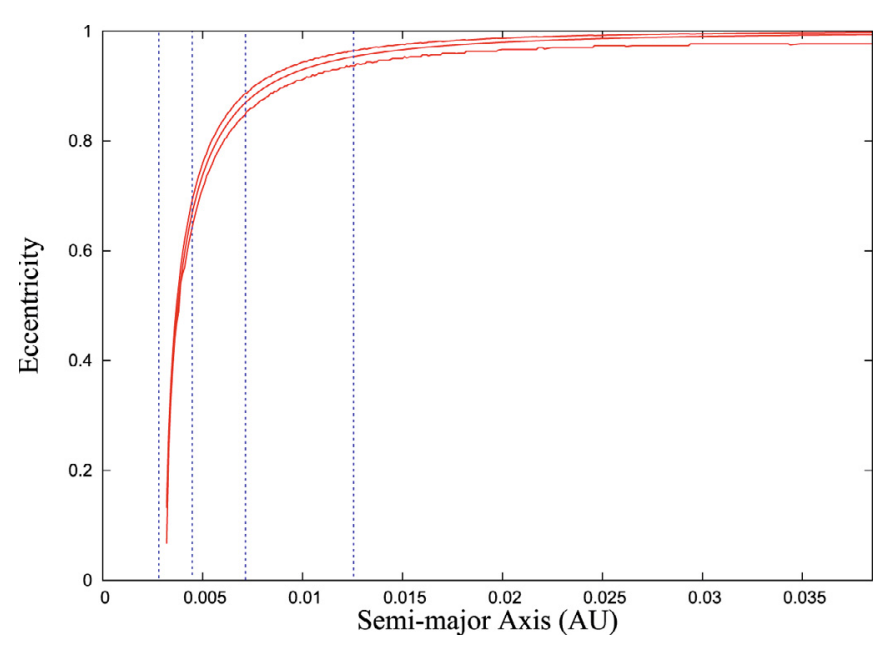

Fig. 13. Location of the evection resonance and its width for Jupiter. The locations in semi-major axis of the Jovian main massive satellites are indicated by vertical lines.

\section{Conclusions}

We have studied the evection resonance using an analytical model extending the computations of Yokoyama et al. (2008). The extended model has different dynamics compared to the 
previous one, mainly in terms of creation of the islands of libration. We have show with an independent method that additional extensions of the model do not alter the predicted dynamics. However, since this analytical model still appears to have limited precision compared to direct numerical integrations due to the chosen averaging method, we resort to surfaces of section to compare them with the model, precisely localize the resonance and study its features. The comparison shows that the extended analytical model, while remaining inaccurate in the localisation of the resonance, reproduces the main features of its dynamics. We determined the appearance of the resonance at the averaged values $a=0.42 r_{\mathrm{h}}$ and $a=0.435 r_{\mathrm{h}}$ for the librations $\alpha=\pi$ and $\alpha=0$ respectively. In addition, we showed that the resonance can be found much closer to the planet due to its oblateness and we indicated its location in terms of semi-major axis and eccentricity. However, important questions about the resonance remain unanswered, for example the dependencies of the resonance on the inclination of orbits and the eccentricity of the perturbing body are of particular interest for escape/capture problems and dynamical studies of outer satellites, and are currently explored. For the same reason, the persistence of the resonance when the motion is subjected to other perturbations, such as massive satellites, planets, or non-gravitational forces, is also investigated and will be presented in a forthcoming paper.

Acknowledgements. The authors would like to thank the anonymous referee for important suggestions and comments.

\section{References}

Alvarellos, J. L. A. 1996, Master's thesis, San José State Univ.

Beaugé, C., \& Nesvorný, D. 2007, AJ, 133, 2537

Breiter, S. 2000, Celest. Mech. \& Dyn. Astr., 77, 201

Brouwer, D., \& Clemence, G. M. 1961, Methods of Celestial Mechanics (New York: Academic Press)

Cincotta, P. M., Giordano, C. M., \& Simó, C. 2003, Physica D, 182, 151

Ćuk, M., \& Burns, J. A. 2004, AJ, 128, 2518

Duriez, L. 2002, Cours de mécanique céleste classique, http://lal. univ-lille1.fr/mecanique_celeste.html

Ferraz-Mello, S. 2007, Canonical Perturbation Theories - Degenerate Systems and Resonance, Astrophysics and Space Science Library (Springer)

Frouard, J., Fouchard, M., \& Vienne, A. 2008, SF2A-2008, 121

Hamilton, D. P., \& Krivov, A. V. 1997, Icarus, 128, 241

Hénon, M. 1969, A\&A, 1, 223

Hénon, M. 1970, A\&A, 9, 24

Moons, M. 1994, Celest. Mech. \& Dyn. Astr., 60, 173

Morbidelli, A. 2002, Modern celestial mechanics : aspects of solar system dynamics (London: Taylor \& Francis)

Murray, C. D., \& Dermott, S. F. 1999, Sol. Sys. Dynamics (Cambridge: Cambridge Univ. Press)

Nesvorný, D., Alvarellos, J. L. A., Dones, L., \& Levison, H. F. 2003, AJ, 126, 398

Saha, P., \& Tremaine, S. 1993, Icarus, 106, 549

Touma, J., \& Wisdom, J. 1998, AJ, 115, 1653

Valtonen, M., \& Karttunen, H. 2006, The Three-body problem (Cambridge: Cambridge University Press)

Yokoyama, T., Santos, M. T., Cardin, G., \& Winter, O. C. 2003, A\&A, 401, 763

Yokoyama, T., Vieira Neto, E., Winter, O. C., Sanchez, D. M., \& de Oliveira Brasil, P. I. 2008, Mathematical Problems in Engineering, doi: 10.1155/2008/251978 